\title{
Essential Fatty Acid Deficiency Depletes Rat Glomeruli of Resident Macrophages and Inhibits Angiotensin II-induced Eicosanoid Synthesis
}

\author{
James B. Lefkowith and George Schreiner* \\ Departments of Medicine and *Pathology, Washington University School of Medicine, St. Louis, Missouri 63110
}

\begin{abstract}
Essential fatty acid (EFA) deficiency exerts a beneficial effect on immune-mediated glomerulonephritis, preventing both the tissue injury and consequent mortality. Because both macrophages and eicosanoids are thought to play pathogenic roles in glomerulonephritis, and because macrophages play an important role in modulating arachidonate metabolism at sites of renal injury, the effects of EFA deficiency on the population of resident glomerular macrophages and on glomerular eicosanoid generation were examined. EFA deficiency led to a striking reduction in the number of resident glomerular macrophages and a corresponding reduction in the number of resident glomerular $\mathrm{Ia}^{+}$cells. This phenomenon was not strain-specific, was not due to a decrease in circulating monocytes, was not a function of changes in cell surface labeling characteristics, and was not restricted to a specific subset of glomeruli. In addition, EFA deficiency affected other areas of the renal cortex: a comparable depletion of interstitial macrophages and $\mathrm{Ia}^{+}$cells was also observed. In conjunction with the decrease in glomerular macrophages seen with the deficiency state, a marked decrease in both basal and angiotensin II-stimulated glomerular eicosanoid production was noted. In contrast to angiotensin II, platelet-activating factor-induced eicosanoid production was not significantly affected by the deficiency state. These changes in glomerular eicosanoid production could not be attributed to changes in glomerular cyclooxygenase or reacylation capacity. Dietary (n-6) fatty acid supplementation, but not (n-3) fatty acid supplementation, reversed both the decrease in glomerular macrophages and the diminished eicosanoid metabolism seen with the deficiency state. Understanding the mechanisms behind the changes in the glomerular microenvironment induced by EFA deficiency may provide a basis for elucidating the protective effect of dietary fatty acid manipulation on immune-mediated glomerulonephritis.
\end{abstract}

\section{Introduction}

Modification of dietary fatty acids has been shown to prolong survival in several animal models of systemic lupus erythema-

Address correspondence to James B. Lefkowith, M.D., Division of Rheumatology, Box 8045, Clinical Sciences Bldg., Washington University School of Medicine, St. Louis, MO 63110.

Received for publication 18 February 1987 and in revised form 14 May 1987.

J. Clin. Invest.

(C) The American Society for Clinical Investigation, Inc.

0021-9738/87/10/0947/10 \$2.00

Volume 80, October 1987, 947-956 tosus. Hurd et al. demonstrated that essential fatty acid (EFA) ${ }^{1}$ deficiency markedly prolonged survival in NZB $\times$ NZW mice (1). Kelley et al. and Prickett et al. showed a similar beneficial effect for diets enriched for (n-3) fatty acids in MRL 1 pr (2) and NZB $\times$ NZW mice (3), respectively. Modification of fatty acid intake appears to be particularly effective in ameliorating the chronic glomerulonephritis associated with the development of autoimmunity, reducing both the incidence of proteinuria and the severity of the attendant histologic changes (1-3). Because renal failure is the principal cause of death in murine lupus (4), the mechanism of the beneficial effect of dietary fatty acid manipulation has been felt to be due to its effects on the development of the glomerulonephritis. The mechanisms by which modulation of fatty acid intake prevent the development of immune-mediated glomerular disease, however, remain ill-defined.

Both glomerular macrophages $(5,6)$ and glomerular eicosanoid production (7) have been implicated pathogenically in glomerulonephritis. The normal rat glomerulus contains a population of macrophages that reside in the mesangium, that are phagocytic and capable of antigen processing, and that can express Ia molecules (8). Both acute and chronic glomerulonephritis in the rat is associated with increased numbers of glomerular macrophages, and these cells appear to play a role in the observed tissue injury $(6,9)$. Additionally, glomerular eicosanoid synthesis has been shown to be increased in glomerulonephritis (both cyclooxygenase [10] and lipoxygenase [11] metabolism), and eicosanoids may mediate some of the attendant functional deficits (7). Evidence also exists to relate these different aspects of renal inflammation: macrophages have been shown to be critically important in modulating arachidonate metabolism at sites of renal injury (12).

Consequently, in an effort to understand the beneficial effects of dietary fatty acid modulation on immune-mediated glomerulonephritis, the present study focuses on the effects of EFA deficiency on both the population of resident glomerular macrophages and on glomerular eicosanoid production. EFA deficiency is shown to lead to a striking depletion of resident glomerular macrophages and to a concurrent diminution of angiotensin II-induced eicosanoid production. Both of these defects are specifically reversed by dietary (n-6) fatty acid supplementation. These findings may provide a basis for understanding the salutary effect of EFA deficiency on immunemediated glomerulonephritis.

\section{Methods}

Reagents and materials. Weanling Sprague-Dawley or Lewis rats were obtained from Charles River Breeding Laboratories, Inc., Wilmington,

1. Abbreviations used in this paper: A II, angiotensin II; EFA, essential fatty acid; EFAD, essential fatty acid deficiency; PAF, platelet-activating factor; PG, prostaglandin; Tx, thromboxane. 
Table I. Effect of Oxygenated Buffer and Collagenase Treatment on Glomerular Eicosanoid Metabolism and Viability

\begin{tabular}{|c|c|c|c|c|c|c|c|}
\hline \multirow[b]{2}{*}{$\begin{array}{l}\text { Incubation } \\
\text { buffer }\end{array}$} & \multirow[b]{2}{*}{$\begin{array}{l}\text { Collagenase } \\
\text { treatment }\end{array}$} & \multicolumn{6}{|c|}{$\mathrm{PGE}_{2}$ production $(\mathrm{ng} / \mathrm{ml})$} \\
\hline & & Basal & $3 \mu \mathrm{m} \mathrm{A} \mathrm{II}$ & Basal & $10 \mu \mathrm{m}$ PAF & $\begin{array}{l}\text { Glomerular } \\
\text { protein }\end{array}$ & $\begin{array}{l}\text { Cells }+ \text { for } \\
\text { trypan blue }\end{array}$ \\
\hline & $U / m l$ & & & & & $\mu g$ & $\%$ \\
\hline PBS & 0 & 0.96 & 1.05 & ND & ND & 641 & 35 \\
\hline KH & 0 & 0.59 & 0.87 & 0.43 & 0.39 & 613 & 7 \\
\hline KH & 10 & 0.68 & 1.30 & 0.33 & 0.72 & 515 & ND \\
\hline KH & 20 & 0.43 & 1.83 & 0.22 & 0.64 & 527 & 2 \\
\hline KH & 50 & 0.39 & 0.97 & 0.25 & 0.48 & 441 & ND \\
\hline
\end{tabular}

Glomeruli were harvested as detailed in Methods and either used untreated or treated with collagenase as indicated. Approximately equal numbers of glomeruli were then incubated for successive 10-min periods in either phosphate-buffered saline (PBS) or oxygenated Kreb'sHenseleit $(\mathrm{KH})$ buffer. Periods of basal $\mathrm{PGE}_{2}$ production were followed by periods of agonist stimulation. $\mathrm{PGE}_{2}$ production was determined by a specific radioimmunoassay. Glomeruli were pelleted after the incubation for protein determination using a fluorescamine assay. Glomerular viability was estimated from enzymatically dissociated cells using trypan blue exclusion.

MA, or Harlan Sprague Dawley, Indianapolis, IN, and fed either a standard lab diet or a fat-free diet purchased from Purina Test Diets, Richmond, IN for at least $8 \mathrm{wk}$. The fatty acid analysis of these diets has been previously published (13). Heneicosanoic acid and its methyl ester, linoleate methyl ester, linolenate methyl ester, angiotensin II (A II), platelet-activating factor (PAF), arachidonic acid, trypsin, and DNAase type I were obtained from Sigma Chemical Co., St. Louis, MO. Fatty acid methyl ester standards for gas chromatography were obtained from Supelco, Inc., Houston, TX. 20:3(n-9) was a gift from Dr. Howard Sprecher, Ohio State University Department of Physiological Chemistry, and its methyl ester was prepared as described (13). Prostaglandin (PG) $E_{2}$, thromboxane (Tx) $B_{2},\left[{ }^{125} I\right] P E_{2},\left[{ }^{125} I\right] T x B_{2}$, and specific antisera to these two eicosanoids were a gift from Dr. Philip Needleman, Washington University, Department of Pharmacology. $\left[{ }^{14} \mathrm{C}\right]$ Arachidonate $(60 \mathrm{mCi} / \mathrm{mmol})$ was purchased from Amersham Corp., Arlington Heights, IL. Antisera for cell labeling (monoclonal anti-rat Ia antibody MAS-028 (Lewis) or MAS-029 (Sprague-Dawley), monoclonal anti-rat leukocyte common antigen antibody OX1HLK, and polyclonal rabbit anti-rat macrophage antiserum) were obtained from Accurate Chemical \& Scientific Corp., Westbury, NY. FITC-labeled rabbit anti-mouse and goat anti-rabbit antisera were purchased from Cappel Laboratories, Cochranville, PA. Collagenase type II was purchased from CooperBiochemical, Inc., Malvern, PA. Tissue culture plates were purchased from Costar, Cambridge, MA. Latex beads $(1 \mu \mathrm{m})$ were purchased from Dow Diagnostics, Indianapolis, IN. Tissue culture media were prepared in the Washington University Cancer Research Center. Organic chemicals were high performance liquid chromatography grade.

Glomerular isolation, incubations, and labeling. Glomeruli were isolated from saline-perfused kidneys using a sieving protocol detailed previously (8). Preparations were typically $>90 \%$ pure. No difference in purity was observed between preparations from normal or EFA-deficient animals.

Initial experiments were performed using previously published protocols for glomerular incubation in which glomeruli are incubated in a physiologic salt solution in the presence or absence of exogenous arachidonate $(7,10,11)$. Products of arachidonate metabolism could be detected in these incubations, but glomeruli failed to respond to hormonal agonists known to stimulate glomerular cell arachidonate metabolism (14-16) (Table I). After incubation, glomeruli were enzymatically dissociated into a suspension of single cells, and glomerular cell viability was determined by exclusion of trypan blue. Glomeruli from these incubations demonstrated extensive cell death (Table I). Substituting an oxygenated buffer was found to reduce cell death extensively, but glomeruli still did not respond to hormonal agonists
(Table I). Because of the possibility that this failure to respond to agonists represented impaired access of the ligand to the interior of the glomerulus, glomeruli were rendered permeable using a mild collagenase exposure. This treatment renders the glomerular mesangium permeable to antibody, permitting in situ labeling of cells in the interior of the glomerulus, but has no effect on overall glomerular architecture except for the removal of Bowman's capsule (8). Incubations with collagenase-permeabilized glomeruli resulted in minimal cell death, a diminished basal arachidonate metabolism relative to nonpermeabilized glomeruli (the elevation of which may have been in part due to ischemia), and unequivocal responses to hormonal agonists (Table I).

As a result of these findings, isolated glomeruli were treated with 20 $\mathrm{U} / \mathrm{ml}$ collagenase type II and $0.01 \mathrm{mg} / \mathrm{ml}$ DNAase for $20 \mathrm{~min}$ at room temperature before use. They were then washed several times with cold oxygenated Kreb's-Henseleit buffer. After collagenase treatment, glomeruli were aliquoted and used in either labeling experiments or in incubations to determine eicosanoid synthesis.

Glomerular incubations were carried out as follows. $\sim 25,000$ glomeruli were suspended in warm oxygenated Kreb's-Henseleit buffer and incubated for successive 10-min periods. After two equilibration periods (during which $0.1 \%$ bovine serum albumin was added to the Kreb's-Henseleit buffer), glomeruli were washed three times, and the basal production of eicosanoids was determined. Glomeruli were then exposed to A II $1 \mu \mathrm{M}$. This concentration of A II had been determined to give a maximal response (Fig. 1). Glomeruli were then washed three

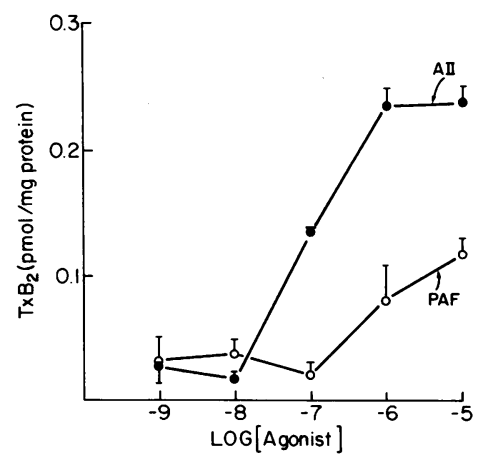

Figure 1. Glomerular synthesis of thromboxane in response to agonists. Glomeruli were isolated and collagenase permeabilized as detailed in Methods. Glomeruli were then incubated with varying concentrations of either A II or $\mathrm{PAF}$. $\mathrm{TxB}_{2}$ production was measured using a specific radioimmunoassay and normalized for glomerular protein content (deter-

mined by a fluorescamine method). The increase over basal is shown and is expressed as the average of three experiments \pm the standard error. 
times and allowed to reequilibrate for one incubation period. Basal production of eicosanoids was subsequently redetermined and the glomeruli were then exposed to $10 \mu \mathrm{M}$ PAF. As with A II, this concentration was determined to give a maximal response (Fig. 1). Glomeruli were again washed three times, the basal production of eicosanoids redetermined a third time, and a final incubation with $10 \mu \mathrm{M}$ arachidonic acid (in oxygenated phosphate-buffered saline) trace-labeled with $\left[{ }^{14} \mathrm{C}\right]$ arachidonate $(1,000 \mathrm{cpm} / 60 \mu \mathrm{l})$ was performed. Incubations were then terminated, and the glomeruli were pelleted and solubilized in $0.62 \mathrm{~N} \mathrm{NaOH}$ for protein determinations. For glomerular lipid analysis experiments the pellet was alternatively subjected to a BlighDyer extraction (17) and the lipids processed as detailed below.

Glomerular production of $\mathrm{PGE}_{2}$ and $\mathrm{TxB}_{2}$ were determined by assaying the Kreb's-Henseleit buffer directly using specific radioimmunoassays detailed previously (18). Sample cross reactivities for the $\mathrm{PGE}_{2}$ antiserum were 6-keto-PGF ${ }_{1 \alpha}, 0.39 \% ; \mathrm{PGF}_{2 \alpha}, 0.04 \%$; and $\mathrm{TxB}_{2}$, $0.003 \%$. Sample cross-reactivities for the $\mathrm{TxB}_{2}$ antiserum were 6-keto$\mathrm{PGF}_{1 \alpha}, 0.007 \%$; $\mathrm{PGE}_{2}, 0.012 \%$; and $\mathrm{PGF}_{2 \alpha}, 0.025 \%$. Glomerular production of eicosanoids was normalized for glomerular protein content, as determined by a fluorescamine assay.

Glomeruli were labeled for leukocyte antigens using a protocol modified from that previously published (19). After collagenase treatment, glomeruli were washed twice in cold phosphate-buffered saline and then placed in one of three primary antibody preparations: (a) monoclonal mouse antibody against rat leukocyte common antigen diluted 1:2 in phosphate-buffered saline; $(b)$ polyclonal rabbit anti-rat macrophage antiserum diluted 1:20 in phosphate-buffered saline; or (c) monoclonal mouse antibody against Lewis Ia antigen (MAS-028) or against a common epitope found in Sprague-Dawley (MAS-029), both diluted $1: 3$ in phosphate-buffered saline. After $20 \mathrm{~min}$ at $4^{\circ} \mathrm{C}$, the glomeruli were washed once, placed in phosphate-buffered saline for $10 \mathrm{~min}$ to permit unbound antibody to diffuse out of the glomerulus, washed again, and placed in a solution of a secondary antibody. Secondary antibodies were either FITC-rabbit anti-mouse antiserum or FITC-goat anti-rabbit antiserum. No labeling was observed with control primary antibody lacking the designated specificities or with secondary antibody exposure only. After washing, the glomeruli were fixed in $2 \%$ paraformaldehyde in phosphate-buffered saline. The labeled cell content of isolated glomeruli was evaluated by microscopic examination with a Universal microscope (Carl Zeiss, Inc., Thornwood, NY). Cells are quantified by focusing through the glomerulus and counting cells as they appear in the plane of focus. In most experiments, 50-100 glomeruli were counted, and the results are expressed as mean \pm SE.

Phagocytic assay of macrophages. Thioglycollate-elicited macrophages were harvested in Hanks' balanced salt solution containing 1\% Hepes buffer. $1 \mathrm{ml}$ containing $2 \times 10^{6}$ cells was placed in duplicate $16-\mathrm{mm}$ wells. Cells were allowed to adhere at $37^{\circ} \mathrm{C}$ for $3 \mathrm{~h}$, washed, layered with $4 \times 10^{7}$ latex beads, and incubated for $1 \mathrm{~h}$. The cells were then washed to remove unbound latex, fixed in $2 \%$ paraformaldehyde in phosphate-buffered saline, and examined by light microsopy. 100 cells from each well were examined. Cells interiorizing three or more particles were scored as positive and the number of particles internalized was recorded. Adherent cell density of control and EFA-deficient macrophages was comparable.

Isolation, culture, and phagocytic assay of glomerular cells. To assay glomerular cells for phagocytosis, glomeruli were dissociated into single cells using a previously detailed procedure (8). In brief, glomeruli were placed in a solution of trypsin $(0.5 \mathrm{mg} / \mathrm{ml})$, collagenase (1 $\mathrm{mg} / \mathrm{ml})$, and DNAase $(0.1 \mathrm{mg} / \mathrm{ml})$ for $30 \mathrm{~min}$ at $37^{\circ} \mathrm{C}$. The glomeruli were then washed in Hanks' balanced salt solution lacking calcium or magnesium and subsequently placed in Hanks' balanced salt solution containing EDTA $2 \mathrm{mM}$ for $20 \mathrm{~min}$ at $37^{\circ} \mathrm{C}$. This preparation was then centrifuged at $50 \mathrm{~g}$ for $10 \mathrm{~min}$ and divided into a sediment and a suspension of single cells. The sediment was additionally subjected to digestion in collagenase $1 \mathrm{mg} / \mathrm{ml}$ for $20 \mathrm{~min}$ at $37^{\circ} \mathrm{C}$. The preparation was then allowed to settle at $1 \mathrm{~g}$ and the supernatant (consisting of single cells) combined with the previous suspension of single cells. Suspensions were then cultured in Hanks' balanced salt solution containing Hepes buffer $1 \mathrm{mM}$ and $1 \%$ bovine serum albumin.

Enzymatically isolated glomerular cells were assayed for phagocytosis as previously detailed (8). Briefly, $1 \times 10^{6}$ cells were cultured for 2 $h$ at $37^{\circ} \mathrm{C}$ in the tissue culture medium described above. After washing, glomerular cells were exposed to latex beads $\left(5 \times 10^{7} / \mathrm{ml}\right)$ for $45 \mathrm{~min}$ at $37^{\circ} \mathrm{C}$ and washed again. Phagocytic cells were defined as those ingesting two or more latex beads. They were quantified by counting cells in 20 randomly selected high-powered fields. Previous studies have established that the adherent phagocytic cells in the rat glomerulus consist solely of marrow-derived resident macrophages $(8,19)$.

Isolation and labeling of interstitial cells. Interstitial cells were obtained by mincing cortex through a sieve with a $250-\mu \mathrm{m}$ pore size. The fragments were weighed, digested in trypsin $0.1 \mathrm{mg} / \mathrm{ml}$ and DNAase $0.1 \mathrm{mg} / \mathrm{ml}$ for $30 \mathrm{~min}$ at $37^{\circ} \mathrm{C}$ (which does not dissociate glomeruli), and then washed. This procedure yielded a suspension of single cells, small clumps of cells, and intact glomeruli. The cells were then labeled with anti-macrophage antiserum or anti-Ia antiserum as previously detailed, washed, and then sandwich-labeled with the appropriate FITC-antibody, also as detailed above. After further washing, the cells were fixed in $1 \%$ paraformaldehyde in phosphate-buffered saline. Positively labeled cells were counted on a per volume basis and normalized for the weight of the cortex predigestion. Positively labeled cells within glomeruli were not counted.

Hepatic and glomerular lipid analysis. Liver lipids from normal and EFA-deficient animals were extracted using a Bligh-Dyer extraction (17). Lipids were then transmethylated (along with heneicosanoic acid as an internal standard), and the resultant fatty acid methyl esters were isolated by thin layer chromatography and characterized by gas chromatography using previously described methods (13).

Glomerular lipids in contrast were first separated into phospholipids using two-dimensional thin layer chromatography, and the fatty acid composition of individual phospholipids determined by transmethylation and gas chromatography as detailed before (13). Heneicosanoic acid methyl ester was used as an internal standard.

Fatty acid repletion experiments. EFA-deficient rats were selectively repleted with either linoleate (18:2[n-6]) or linolenate (18:3[n-3]) methyl ester as follows. Animals were injected with $1 \mathrm{~g} / \mathrm{kg}$ i.p. of the fatty acid methyl ester once daily $5 \mathrm{~d}$ a week for a period of $1 \mathrm{mo}$. Animals were subsequently used for glomerular incubations, glomerular labeling experiments, and liver and glomerular lipid analyses.

\section{Results}

Validation of EFA deficiency. Rats fed an EFA-deficient diet exhibited the characteristic dermatitis and mild growth retardation (average, $10 \%$ ). To validate the deficiency state biochemically, fatty acid analysis was performed on both liver and glomerular lipids. Liver lipids showed a $>90 \%$ depletion of arachidonate and a 20:3(n-9) to arachidonate ratio of 3.71 with EFA deficiency (Table II). A 20:3(n-9) to arachidonate ratio of $>0.4$ is the biochemical criterion for EFA deficiency (20).

Glomerular lipids were fractionated into individual phospholipids before fatty acid analysis. Glomerular phospholipid arachidonate was also depleted with EFA deficiency, though less so than liver arachidonate (Table III). All four classes of phospholipids showed decreased arachidonate with EFA deficiency, ranging from a $71 \%$ decrease in phosphatidylcholine to a 24\% decrease in phosphatidylserine. The 20:3(n-9) to arachidonate ratios seen in the individual phospholipids also varied widely, ranging from 3.00 in phosphatidylcholine to 1.56 in phosphatidylserine. All these values, however, exceeded the cutoff value of 0.4 . Thus both the liver and the tissue of inter- 
Table II. Liver Lipid Fatty Acid Analysis

\begin{tabular}{llll}
\hline & AA & $20: 3(\mathrm{n}-9)$ & $20: 3(\mathrm{n}-9) / \mathrm{AA}$ \\
\hline & mol \% & mol \% & mol \% \\
Control $(n=7)$ & $15.0 \pm 0.9$ & $\mathrm{ND}$ & 0.00 \\
EFAD $(n=5)$ & $1.4 \pm 0.3^{*}$ & $5.2 \pm 0.9^{*}$ & 3.71 \\
EFAD $+18: 2(n=3)$ & $5.1 \pm 0.9^{\ddagger}$ & $0.2 \pm 0.0^{\ddagger}$ & 0.04 \\
EFAD $+18: 3(n=4)$ & $0.5 \pm 0.2^{\S}$ & $0.3 \pm 0.2^{\ddagger}$ & 0.60
\end{tabular}

Liver lipids from control, EFA-deficient, EFA-deficient/linoleatesupplemented, and EFA-deficient/linolenate-supplemented animals were extracted using a Bligh-Dyer extraction (17). The constituent fatty acids were then transmethylated, isolated by thin layer chromatography, and characterized and quantified using gas chromatography. $P<0.01$ for one-way analysis of variance on both AA and 20:3(n-9) groups. Means were compared using Student's $t$ test. $* P<0.01$; EFA-deficient vs. control.

${ }^{\ddagger} P<0.01$; EFAD + 18:2 or EFAD + 18:3 vs. EFA-deficient.

${ }^{8} P<0.05 ;$ EFAD $+18: 3$ vs. EFA-deficient. Abbreviations: AA, arachidonate; EFAD + 18:2, linoleate-supplemented EFA-deficient animals; EFAD + 18:3, linolenate-supplemented EFA-deficient animals; ND, none detected.

est, the glomeruli, exhibited the characteristic biochemical changes of the deficiency state.

Effects of EFA deficiency on resident glomerular macrophages. The effects of the deficiency state on the macrophage content of glomeruli were determined in two strains of rats (outbred Sprague-Dawley and inbred Lewis) using a fluorescence-labeling technique to quantify macrophages on a per glomerulus basis. Compared with controls, EFA-deficient glomeruli exhibited a marked depletion of resident macrophages $(\sim 70-80 \%$, Fig. $2 A$ and $3 A$ ). When glomeruli were labeled for the subset of macrophages that express the Ia antigen and were thus immunologically competent, a comparable depletion of $\mathrm{Ia}^{+}$cells was also seen with the deficiency state (Figs. $2 B$ and $3 B$ ). Representative pictures of glomeruli from normal and EFA-deficient rats stained for macrophages or $\mathrm{Ia}^{+}$cells are shown in Fig. 4.

These differences in glomerular macrophage content could not be explained by a more generalized depletion of circulating leukocytes or monocytes by EFA deficiency. Circulating leukocyte counts were $12,811 \pm 2,475 / \mathrm{mm}^{3}$ in controls and $12,833 \pm 808 / \mathrm{mm}^{3}$ in EFA-deficient animals, respectively (mean $\pm \mathrm{SE}, n=6)$. Circulating monocyte counts were $2,241 \pm 944 / \mathrm{mm}^{3}$ in controls and $1,866 \pm 401 / \mathrm{mm}^{3}$ in EFA-deficient animals, respectively (mean $\pm \mathrm{SE}, n=6$ ). These data corroborate those of other investigators (21) and establish that peripheral leukocyte counts (particularly monocyte counts) are unaffected by the deficiency state.

The possibility that EFA deficiency might have affected the expression of macrophage cell surface antigens, thus leading to a decrease in macrophage labeling rather than an actual decrease in glomerular macrophage number, was also considered. In consequence, a functional assay for resident glomerular macrophages, phagocytosis $(8,19)$, was used to quantify these cells. Initial experiments determined the effect of EFA deficiency on macrophage phagocytosis. EFA-deficient macrophages exhibited comparable, or slightly enhanced, phagocytosis relative to control. $95 \%$ of adherent EFA-deficient macrophages were phagocytic compared with $63 \%$ of the control cells. EFA-deficient macrophages were also highly phagocytic. $100 \%$ of the positive cells ingested 50 beads or more compared with $76 \%$ for positive control cells. These results are in accordance with previous findings that, in mice, macrophage phagocytosis of zymosan is unaffected by EFA deficiency (18). Equal numbers of glomeruli from control and EFA-deficient animals were then enzymatically dissociated into a single cell suspension, placed in short-term tissue culture, and challenged with latex beads. The number of phagocytic cells per highpowered field was subsequently determined. Employing this functional criterion, a marked decrease in glomerular macrophage number with EFA-deficiency was again noted (Fig. 5) and was comparable with that seen with the fluorescence labeling technique (Fig. 2). These data thus validate those obtained with the fluorescence labeling technique and demonstrate conclusively that resident glomerular macrophage number is decreased in EFA deficiency.

To examine whether a subset of glomeruli were preferentially affected by EFA deficiency or whether glomeruli were uniformly affected, the distribution of macrophages in glomeruli from both EFA-deficient and control Lewis rats was determined. As shown in Fig. 6, control glomeruli exhibited a

Table III. Glomerular Phospholipid Fatty Acid Analysis

\begin{tabular}{|c|c|c|c|c|c|c|c|}
\hline & \multicolumn{4}{|c|}{ Phospholipid arachidonate } & \multicolumn{3}{|c|}{ Phospholipid 20:3(n-9) and 20:3(n-9)/arachidonate ratios } \\
\hline & Control & & EFAD & & EFAD & & \\
\hline & $\mathrm{mol} \%$ & nmol/mg protein & $\mathrm{mol} \%$ & nmol/mg protein & $\mathrm{mol} \%$ & nmol/mg protein & $20: 3(n-9) /$ arachidonate \\
\hline PC & $12.7 \pm 1.1$ & $10.5 \pm 1.6$ & $3.7 \pm 0.6^{*}$ & $3.9 \pm 1.0^{\ddagger}$ & $11.1 \pm 0.3^{*}$ & $9.9 \pm 1.7^{*}$ & 3.00 \\
\hline PI & $25.9 \pm 4.3$ & $2.9 \pm 0.6$ & $8.2 \pm 1.6^{*}$ & $1.1 \pm 0.2^{\ddagger}$ & $22.8 \pm 1.6^{*}$ & $2.2 \pm 0.2^{*}$ & 2.78 \\
\hline PE & $34.5 \pm 4.4$ & $22.9 \pm 4.8$ & $13.1 \pm 1.6^{*}$ & $8.7 \pm 1.7^{\ddagger}$ & $24.5 \pm 1.6^{*}$ & $15.6 \pm 1.4^{*}$ & 1.87 \\
\hline PS & $8.7 \pm 1.4$ & $3.2 \pm 0.8$ & $6.6 \pm 0.2$ & $3.0 \pm 0.8$ & $10.3 \pm 1.0^{*}$ & $4.3 \pm 2.1^{\ddagger}$ & 1.56 \\
\hline
\end{tabular}

Glomerular lipids from control and EFA-deficient animals were extracted and separated into their constituent phospholipids using two-dimensional thin layer chromatography. The fatty acids of individual phospholipids were then transmethylated, and characterized and quantified using gas chromatography. Means were compared with Student's $t$ test. ${ }^{*} P<0.01$; EFA-deficiency vs. control ( $n=4$, all groups). $\ddagger P<0.05$. Abbreviations: PC, phosphatidylcholine; PE, phosphatidylethanolamine; PI, phosphatidylinositol; PS, phosphatidylserine. No 20:3(n-9) was detected in control samples. 


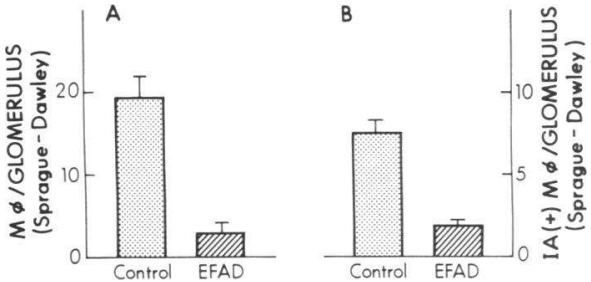

Figure 2. Effect of EFA deficiency on glomerular macrophage and $\mathrm{Ia}^{+}$cell number in Sprague-Dawley outbred rats. Glomeruli were isolated using a sieving protocol, permeabilized with collagenase and labeled with monoclonal antibodies using a double-antibody fluorescence technique as detailed in Methods. Macrophages were detected using an anti-leukocyte common antigen primary antibody. Between 50 and 100 glomeruli were examined per animal, and the number of positive staining cells was expressed on a per glomerulus basis. The average \pm standard error are shown for a pool of glomeruli from two animals. The experiment was repeated three times with identical results. $(A)$ Macrophages per glomerulus, EFA deficiency vs. control. (B) $\mathrm{Ia}^{+}$cells per glomerulus, EFA deficiency vs. control.

roughly mean distribution with respect to macrophage content, peaking with an average representation of eight to nine macrophages per glomerulus; no glomeruli were entirely devoid of resident macrophages. In contrast, the entire population of glomeruli demonstrated a shift to the left with EFA deficiency, indicating no selectivity in how the deficiency state affected glomeruli. $>40 \%$ of EFA-deficient glomeruli had virtually no detectable macrophages, while $>70 \%$ had three or fewer.

Additional experiments addressed the issue of whether the depletion of macrophages in the renal cortex seen with EFA deficiency was specific to the glomerulus. Extraglomerular renal cortex was enzymatically dissociated into a single cell suspension and subsequently labeled for either macrophages or $\mathrm{Ia}^{+}$cells. These experiments demonstrated that EFA deficiency also led to a marked depletion of interstitial macrophages and $\mathrm{Ia}^{+}$cells (Fig. 7). Thus the depletion of macrophages seen with the deficiency state was a generalized phenomenon with respect to the renal cortex: resident macrophages were depleted from both the glomeruli and the interstitium.

Effects of EFA deficiency on glomerular eicosanoid production. Glomerular cells are known to synthesize eicosanoids basally and in response to a variety of agonists (14-16). The effects of EFA deficiency on glomerular eicosanoid production were thus determined by measuring both the basal production of $\mathrm{PGE}_{2}$ and $\mathrm{TxB}_{2}$ and the production of these eicosanoids in response to two potentially important agonists with respect to glomerulonephritis, A II (22) and PAF (23).

Glomerular basal production of $\mathrm{PGE}_{2}$ and $\mathrm{TxB}_{2}$ was suppressed by the deficiency state. An 50\% decrease in the basal synthesis of these eicosanoids was seen with EFA deficiency (Table IV). An even more striking effect was seen when A II-stimulated eicosanoid metabolism was examined. Control glomeruli responded to A II stimulation with a three- to fivefold increase in both $\mathrm{PGE}_{2}$ and $\mathrm{TxB}_{2}$ production (Table IV). EFA-deficient glomeruli exhibited a markedly decreased generation of these eicosanoids in response to $\mathrm{A}$ II: $\mathrm{PGE}_{2}$ and $\mathrm{TxB}_{2}$ production were decreased by $85 \%$ relative to control (Table IV).
EFA deficiency, in contrast, did not significantly affect the elaboration of eicosanoids in response to the other agonist utilized, PAF. In normal glomeruli, PAF led to a doubling of $\mathrm{PGE}_{2}$ and $\mathrm{TxB}_{2}$ production (Table IV). The production of these eicosanoids in response to this agonist by EFA-deficient glomeruli was modestly decreased relative to control glomeruli (Table IV).

The observed differences in eicosanoid production between EFA-deficient and control glomeruli could not be attributed to differences in glomerular cyclooxygenase capacity. The conversion of exogenous arachidonate to both $\mathrm{PGE}_{2}$ and $\mathrm{TxB}_{2}$ by EFA-deficient glomeruli was actually severalfold greater than that seen with control glomeruli (Fig. 8). Glomerular eicosanoid production assessed using exogenous arachidonate was thus strikingly different than glomerular eicosanoid production assessed using agonists causing the release of endogenous arachidonate. EFA-deficient glomeruli were markedly deficient in the production of eicosanoids in response to A II (but not PAF). In contrast, EFA-deficient glomeruli actually produced greater quantities of eicosanoids relative to control when provided with exogenous substrate.

The differences in agonist-stimulated eicosanoid production between EFA-deficient and control glomeruli also could not be explained by differences in glomerular reacylation ability which might affect the availability of free fatty acid for metabolism. The reuptake of labeled exogenous arachidonate was the same in EFA-deficient and control glomeruli: 95 and 92\% in EFA-deficient glomeruli and 94 and $95 \%$ in control glomeruli.

Effects of selected fatty acid repletion on the changes in glomerular macrophages and eicosanoid metabolism seen with EFA deficiency. To determine whether the changes in glomerular macrophage number and glomerular prostaglandin production were specifically a function of (n-6) or (n-3) fatty acids, and to assess the reversibility of these changes, we performed a series of experiments in which EFA-deficient animals were supplemented with either linoleate methyl ester or linolenate methyl ester. As shown in Table II, supplementation with linoleate restored liver arachidonate levels to more normal levels and markedly decreased both the 20:3(n-9) level and the $20: 3(n-9)$ to arachidonate ratio. Linolenate supplementation of EFA-deficient animals, in contrast, further decreased arachidonate levels (Table II). The percentage of 20:3(n-9), as well as the 20:3(n-9) to arachidonate ratio, however, were suppressed with linolenate supplementation. Linolenate supplementation also increased liver (n-3) fatty acids, particularly

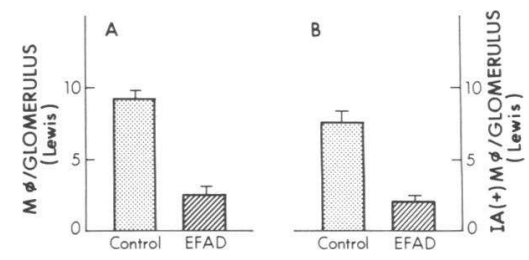

Figure 3. Effect of EFA deficiency on glomerular macrophage and $\mathrm{Ia}^{+}$ cell number in Lewis inbred rats. Glomeruli were isolated, processed, and examined as detailed in the legend to

Fig. 1 except that macrophages were detected using an anti-macrophage antibody. Average cell number \pm standard error are shown for a pool of glomeruli from two animals. The experiment was repeated three times with identical results. $(A)$ Macrophages per glomerulus, EFA deficiency vs. control. $(B) \mathrm{Ia}^{+}$cells per glomerulus, EFA deficiency vs. control. 

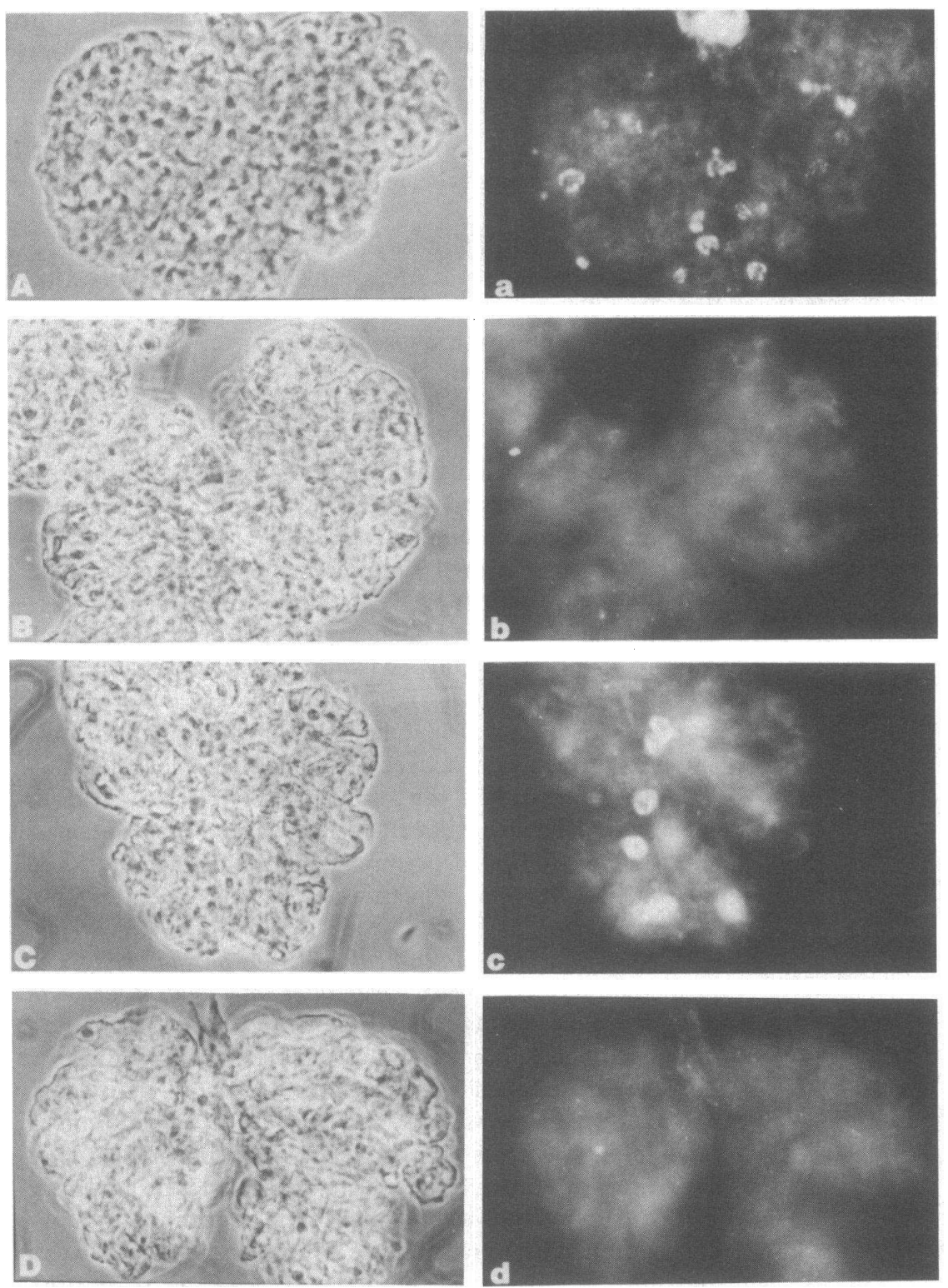

Figure 4. Representative light and fluorescent photomicrographs of labeled EFA-deficient and control glomeruli from Lewis rats. Glomeruli were isolated, processed, and examined as detailed in the legend to Fig. 1. ( $A$ and $C$ ) Light photomicrographs of control glomeruli. $(B$ and $D)$ Light photomicrographs of EFA-deficient glomeruli. $(a$ and $b$ ) Fluorescent photomicrographs of control and EFA-deficient glomeruli stained for macrophages, respectively. $(c$ and $d$ ) Fluorescent photomicrophages of control and EFA-deficient glomeruli stained for Ia antigen, respectively. Artifactual staining of the glomerular hilus (see $a$ ) was occasionally seen and was regarded as nonspecific, in that it was observed when glomeruli were stained with secondary antibody only. eicosapentaenoic acid (20:5[n-3]), which was not present in control animals $(2.5 \pm 1.2 \%$ in linolenate-supplemented EFAdeficient animals).

The decrease in glomerular phospholipid arachidonate content was more completely reversed by linoleate supple-

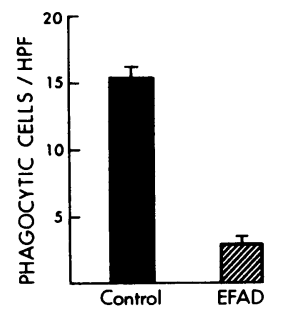

Figure 5. Phagocytic cell content of EFA-deficient and control glomeruli. EFA-deficient and control glomeruli were dissociated into a single cell preparation using a digestion protocol detailed in Methods. Single cell preparations were then challenged with latex beads $\left(5 \times 10^{7} / \mathrm{ml}\right)$. The number of phagocytic cells per high-powered field was subsequently quantified in 50 fields and is expressed as an average \pm standard error. A representative experiment is shown.

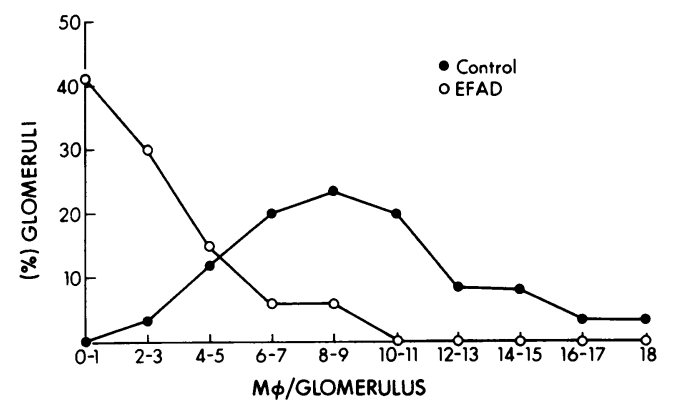

Figure 6. Frequency distribution of the number of macrophages per glomerulus in EFA-deficient and control glomeruli. The number of macrophages per glomerulus in EFA-deficient and control glomeruli was determined as detailed in the legend to Fig. 1. The data from three animals were pooled $(\sim 100$ glomeruli were examined per animal) and are expressed as percent of total glomeruli examined vs. macrophages per glomerulus. 

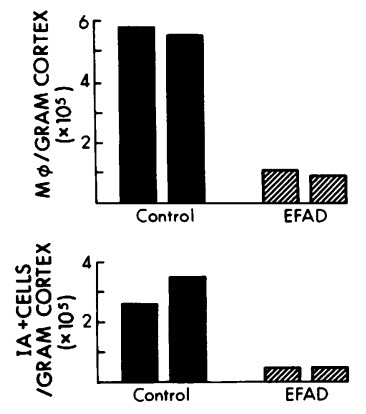

nique detailed in Methods. The number of positive staining cells per gram of starting material was calculated. Positive-staining cells within glomeruli were not counted. Two representative control and EFA-deficient animals are shown.

mentation in comparison to the liver. Levels of glomerular phospholipid arachidonate in linoleate-supplemented EFAdeficient animals were virtually normal (Table $\mathrm{V}$ ). Levels of phospholipid 20:3(n-9), as well as 20:3(n-9) to arachidonate ratios, were also suppressed by linoleate supplementation (Table V). Linolenate supplementation of EFA-deficient animals, in contrast did not restore levels of glomerular phospholipid arachidonate, but did decrease levels of phospholipid 20:3(n-9) and suppress 20:3(n-9) to arachidonate ratios (Table V). Levels of glomerular phospholipid (n-3) fatty acids (particularly 20:5[n-3]) were also increased (data not shown).

Numbers of glomerular macrophages were subsequently quantified in the four different groups of animals: control, EFA-deficient, EFA-deficient/linoleate-supplemented, and EFA-deficient/linolenate-supplemented animals. As shown in Fig. 9, levels of macrophages were increased to approximately twice normal levels by linoleate supplementation. Linolenate supplementation did not increase levels of macrophages in the glomerulus above that seen in EFA deficiency (Fig. 9).

The eicosanoid metabolism of glomeruli from these four groups of animals was determined in parallel to the labeling experiments. As noted in Table VI, glomeruli from linoleatesupplemented EFA-deficient animals exhibited an increased basal production of $\mathrm{PGE}_{2}$ and $\mathrm{TxB}_{2}$ to levels roughly two- to threefold greater than control. A II-stimulated eicosanoid pro-
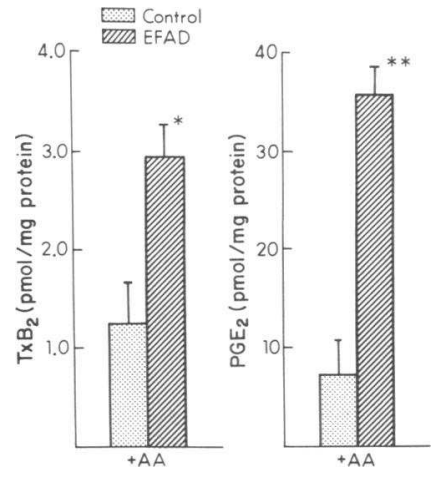

Figure 8. Conversion of exogenous arachidonate to $\mathrm{PGE}_{2}$ and $\mathrm{TxB}_{2}$ by control and EFAdeficient glomeruli. Glomeruli were isolated and permeabilized with collagenase as detailed in Methods. Isolated glomeruli were then incubated with arachidonate $10 \mu \mathrm{M}$. $\mathrm{PGE}_{2}$ and $\mathrm{TxB}_{2}$ production were measured using specific radioimmunoassays, and production was normalized for glomerular protein content (determined by a fluorescamine method). The increase over basal is shown and is expressed as the average of three experiments \pm the standard error. EFA-deficient values were compared to control values using Student's $t$ test. Significance levels are ${ }^{*} P<0.05,{ }^{* *} P$ $<0.01$.

duction by these glomeruli, however, was simply restored to control levels. Glomeruli from linolenate-supplemented EFAdeficient animals exhibited an even lower basal production of eicosanoids relative to EFA-deficient glomeruli (Table VI). These glomeruli also exhibited a suppressed response to A II stimulation comparable to EFA-deficient glomeruli.

In sum, both the decrease in glomerular macrophage number and the decrease in glomerular eicosanoid production induced EFA deficiency were reversible, covariant, and specifcally a function of the presence of $(n-6)$ rather than $(n-3)$ fatty acids.

\section{Discussion}

This study demonstrates two novel effects of EFA deficiency on the glomerulus. One is a marked depletion in the number of resident mesangial macrophages without a commensurate decrease in circulating monocytes. The other is a selective effect on agonist-induced glomerular eicosanoid production. These two effects of EFA deficiency are reversible with (n-6) but not (n-3) fatty acids supplementation and appear to vary in parallel.

Table IV. Glomerular Eicosanoid Synthesis: EFA Deficiency vs. Control

\begin{tabular}{|c|c|c|c|c|c|c|}
\hline & \multicolumn{3}{|c|}{$\mathrm{PGE}_{2}$ production (pmol/mg protein) } & \multicolumn{3}{|c|}{$\mathrm{TxB}_{2}$ production (pmol/mg protein) } \\
\hline & Control & EFAD & $\begin{array}{l}\% \text { decrease } \\
\text { from control }\end{array}$ & Control & EFAD & $\begin{array}{l}\% \text { decrease } \\
\text { from control }\end{array}$ \\
\hline Basal & $1.32 \pm 0.15$ & $0.77 \pm 0.12^{*}$ & 42 & $0.174 \pm 0.017$ & $0.065 \pm 0.020^{\ddagger}$ & 63 \\
\hline \multicolumn{7}{|c|}{ A II $(1 \mu M)$} \\
\hline Total & $6.70 \pm 1.33$ & $1.29 \pm 0.29^{*}$ & 81 & $0.593 \pm 0.039$ & $0.129 \pm 0.030^{\ddagger}$ & 78 \\
\hline Net & $5.56 \pm 0.97$ & $0.81 \pm 0.18^{\ddagger}$ & 85 & $0.420 \pm 0.042$ & $0.064 \pm 0.015^{\ddagger}$ & 85 \\
\hline \multicolumn{7}{|c|}{ PAF $(10 \mu M)$} \\
\hline Total & $2.24 \pm 0.57$ & $1.65 \pm 0.32$ & 26 & $0.369 \pm 0.107$ & $0.209 \pm 0.048$ & 43 \\
\hline Net & $1.27 \pm 0.21$ & $0.92 \pm 0.35$ & 28 & $0.194 \pm 0.069$ & $0.129 \pm 0.059$ & 34 \\
\hline
\end{tabular}

Glomeruli from control and EFA-deficient animals were isolated and incubated as detailed in Methods. Glomerular $\mathrm{PGE}_{2}$ and TxB $\mathrm{B}_{2}$ synthesis was determined by specific radioimmunoassay and normalized for the amount of glomerular protein present. Net synthesis represents total minus basal synthesis. Means were compared with Student's $t$ test. ${ }^{*} P<0.05 ;{ }^{\ddagger} P<0.01$; EFA-deficient vs. control ( $n=3$, all groups). 
Table V. Glomerular Phospholipid Fatty Acid Analysis Post Linoleate and Linolenate Supplementation

\begin{tabular}{llllll}
\hline \multicolumn{4}{l}{ 20:3(n-9)/Arachidonate (calculated ratio) } \\
\cline { 2 - 6 } & Control & EFAD & EFAD + 18:2 & EFAD + 18:3 \\
\hline PC & ND/16.2(0.0) & $11.0 / 2.0(5.5)$ & $0.1 / 15.8(0.0)$ & $1.5 / 2.3(0.6)$ \\
PI & ND/32.2(0.0) & $19.6 / 4.2(4.6)$ & $5.5 / 24.3(0.2)$ & ND/15.0 (0.0) \\
PE & ND/30.8(0.0) & $28.7 / 8.6(3.3)$ & $1.5 / 35.7(0.0)$ & $4.0 / 11.5(0.3)$ \\
PS & ND/12.0(0.0) & $13.0 / 6.3(2.1)$ & $0.9 / 10.6(0.1)$ & $1.6 / 3.1$ & $(0.5)$
\end{tabular}

Glomerular lipids from control, EFA-deficient, EFA-deficient/linoleate-supplemented and EFA-deficient/linolenate-supplemented animals were extracted and separated by two-dimensional thin layer chromatography. The constituent fatty acids of individual phospholipids were transmethylated and quantified by gas chromatography. The analysis was performed with a pool of glomeruli from two animals. Abbreviations: PC, phosphatidylcholine; PE, phosphatidylethanolamine; PI, phosphatidylinositol; PS, phosphatidylserine; EFAD $+18: 2$, linoleate-supplemented EFA-deficient; EFAD + 18:3, linolenate-supplemented EFA-deficient; ND, none detected.

The changes in eicosanoid production seen with EFA deficiency are established by using a newly developed method for examining the endogenous production of glomerular eicosanoids. Isolated glomeruli are first permeabilized with collagenase and then incubated in an oxygenated buffer. This protocol minimized cell death and makes it possible to determine agonist-stimulated eicosanoid metabolism in whole glomeruli without adding arachidonate. Thus this new method has the advantage of determining the actual production of eicosanoids, rather than just the potential for the production of eicosanoids as determined with the usual method of adding arachidonate. $\mathrm{PGE}_{2}$ and $\mathrm{TxB}_{2}$ production, both basally and in response to A II stimulation, are shown to be markedly reduced by the deficiency state by this new method. The addition of exogenous arachidonate, in contrast, demonstrates an enhanced conversion of arachidonate in EFA deficiency, indicating that modulation of eicosanoid synthesis is not occurring at the level of cyclooxygenase activity. The use of exogenous arachidonate alone to determine glomerular eicosanoid metabolism thus would have obscured the changes induced by the deficiency state. In fact, the severalfold increase in cyclooxygenase activity seen in EFA deficiency makes even more striking the virtual complete suppression of A II-induced eicosanoid synthesis seen in the deficiency state.

The observation that EFA deficiency affects A II-induced eicosanoid synthesis more than that induced by PAF is similar to a previously described phenomenon in isolated perfused EFA-deficient kidneys. EFA deficiency was shown to decrease markedly A II-stimulated $\mathrm{PGE}_{2}$ production (13). In contrast, ischemia-induced $\mathrm{PGE}_{2}$ production was unaffected (13). This difference was attributed to the findings that EFA deficiency preferentially depletes phosphatidylinositol of arachidonate (13), and that this phospholipid pool may be the source of arachidonate in response to A II stimulation (24). Ischemia, in comparison to A II, is a relatively less specific stimulus for fatty acid release (25). Studies also suggest that PAF is a less specific stimulus than A II and induces the release of arachidonate from several phospholipids (15). This difference may explain the differential effect of EFA deficiency on these two agonists.
Manipulation of dietary fatty acids (both EFA deprivation and (n-3) fatty acid supplementation) has been shown to be remarkably protective against the lethal glomerulonephritis that develops in murine lupus (1-3). The basic explanations behind this phenomenon, however, have not been established. Suppression of autoimmunity has been suggested as a possible mechanism of the protective effect (2). Available studies, however, would seem to contradict this hypothesis. No unequivocal effect of EFA deficiency has been established with respect to systemic immunity, either humoral or cellular (reviewed in reference 26). In fact, some studies have shown an increase in both humoral (27) and cell-mediated immunity (28). Moreover, dietary fatty acid manipulation has been shown to exert a protective effect despite the fact that anti-dsDNA autoantibodies are not suppressed (2). Additionally, institution of dietary fatty acid manipulation has been shown to be protective even when started after the inception of renal disease (29). The data presented in this study would suggest an entirely different possibility: that modulation of the glomerular microenvironment (i.e., glomerular macrophage number and eicosanoid production) may contribute to the renal-sparing effects of dietary fatty acid restriction in lupus glomerulonephritis.

The contributing role of macrophages in the evolution of glomerulonephritis has received increasing emphasis. Studies have established that rat glomeruli contain a resident population of macrophages that are derived from the bone marrow, that are phagocytic, and that express Ia antigens (19). Such cells are immunologically competent and are capable of presenting antigen to appropriately sensitized lymphocytes (8). Their relative depletion from the kidney and virtual absence from approximately half of the glomeruli as seen in EFA deficiency could conceivably protect against the initiation of an in situ immune reaction. Additionally, the same effects inhibiting the migration of monocytes into the glomerulus in EFA deficiency could affect the mobilization of leukocytes into the glomerulus in the context of glomerular inflammation. This influx of leukocytes is causally associated with the glomerular damage in experimental glomerulonephritides $(5,6)$. In support of this contention, we have preliminary evidence that EFA deficiency largely abrogates the influx of macrophages into the glomerulus in response to inflammatory stimuli (30).

The modulation of glomerular arachidonate metabolism induced by EFA deficiency could be equally significant in ameliorating immune-mediated glomerular inflammation.

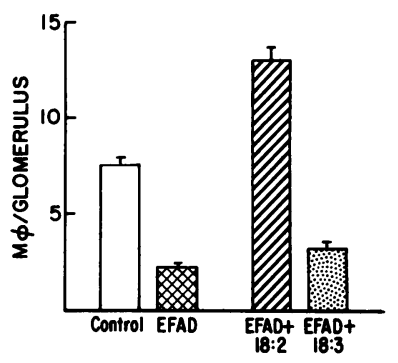

Figure 9. Effect of linoleate and linolenate supplementation of EFA-deficient animals on glomerular macrophage number. EFAdeficient animals were repleated with either linoleate or linolenate. Glomeruli from these animals were then examined as detailed in the legend to Fig. 1 and the number of macrophages per glomerulus calculated. Parallel controls

with EFA-deficient and control animals were performed. The data were derived from a pool of glomeruli from two animals and are expressed as an average \pm standard error. Abbreviations: EFAD

+ 18:2, EFA-deficient/linoleate-supplemented; EFAD + 18:3, EFAdeficient/linolenate-supplemented. 
Table VI. Glomerular Eicosanoid Synthesis in Control, EFA-deficient, EFA-deficient/Linoleate-supplemented, and EFA-deficient/Linolenate-supplemented Animals

\begin{tabular}{|c|c|c|c|c|c|c|c|c|}
\hline & \multicolumn{4}{|c|}{$\mathrm{PGE}_{2}$ production (pmol/mg protein) } & \multicolumn{4}{|c|}{$\mathrm{TxB}_{2}$ production (pmol/mg protein) } \\
\hline & Control & EFAD & EFAD + 18:2 & EFAD $+18: 3$ & Control & EFAD & EFAD + 18:2 & EFAD + 18:3 \\
\hline Basal & $1.97,1.20$ & $1.02,1.05$ & $4.78,5.67$ & ND & $0.423,0.278$ & $0.109,0.101$ & $0.813,0.511$ & $0.041,0.056$ \\
\hline A II $1 \mu \mathrm{M}$ & $7.51,6.65$ & $2.43,2.43$ & $6.54,7.93$ & $0.76,1.57$ & $1.213,1.082$ & $0.248,0.226$ & $1.080,0.979$ & $0.183,0.245$ \\
\hline
\end{tabular}

Glomeruli from each of the four groups of animals were isolated and incubated as detailed in Methods. Eicosanoid synthesis was normalized for the amount of glomerular protein present. A II-stimulated production is expressed as the increase over basal production. Abbreviations: EFAD + 18:2, EFA-deficient/linoleate-supplemented; EFAD + 18:3, EFA-deficient/linolenate-supplemented; ND, none detected. Because $n$ $=2$ for all groups, both replicates are expressed.

Studies have established an enhanced glomerular production of eicosanoids in glomerulonephritis $(10,11,31)$ and have shown that eicosanoids may play a role in the pathophysiologic changes that occur $(7,31)$. Studies also suggest a role for A II in glomerulonephritis and suggest that this autocoid may be an agonist in vivo for eicosanoid production (22). The inhibition of basal as well as A II-stimulated eicosanoid production seen with EFA deficiency might therefore ameliorate the altered glomerular physiology in nephritis.

The observation that EFA deficiency affects both glomerular macrophage representation and arachidonate metabolism in concert, and that both of these changes are reversed concomitantly with (n-6) fatty acid supplementation suggests that these phenomena may be causally interrelated. Independent lines of observation would tend to support this conjecture. Macrophages have been shown to affect dramatically the arachidonate metabolism of a variety of mesenchymal cells (32), including mesangial cells (33). This interaction occurs via the elaboration of soluble factors, most notably interleukin 1 (32). Mononuclear cell factor(s) have been shown to increase both mesenchymal cell basal and hormonal agonist-stimulated $\mathrm{PGE}_{2}$ production up to 100 -fold (34). The absence of macrophages from EFA-deficient glomeruli thus may explain the decrease in A II-stimulated eicosanoid production because mesangial cells are the only glomerular cell to express receptors for A II (35). This hypothesis is supported by studies that demonstrate that monocyte depletion prevents the influx of macrophages, as well as the markedly enhanced agonist-stimulated eicosanoid production, seen in a model of experimental renal inflammation, hydronephrosis (12).

The observation that linolenic acid supplementation did not reverse the decrease in glomerular macrophages or the decrease in eicosanoid synthesis seen with EFA deficiency suggests a parallel between the mechanisms underlying the protective effect of EFA deficiency and the protective effect of (n-3) fatty acid-enriched diets. In fact there are many similarities between the biochemical effects of (n-3) fatty acid enrichment and EFA deficiency with respect to eicosanoid production. Eicosapentaenoic acid, the (n-3) fatty acid which is augmented most dramatically by linolenic supplementation, is a poor substrate for cyclooxygenase but does inhibit prostaglandin formation from arachidonate by competing for cyclooxygenase (36). With respect to lipoxygenase metabolism, eicosapentaenoic acid is converted to leukotriene $A_{5}$ and subsequently to leukotrienes $C_{5}$ and $B_{5}(37)$. Leukotriene $A_{5}$ is inefficiently converted to leukotriene $B_{5}$, and inhibits the for- mation of leukotriene $B_{4}$, possibly by covalently coupling to leukotriene A hydrolase (38). In comparison, 20:3(n-9), which accumulates in the deficiency state, is also not a particularly good substrate for cyclooxygenase (39). EFA deficiency, however, does decrease prostaglandin production in response to certain stimuli (e.g., A II), probably by depleting arachidonate from selected phospholipid pools rather than by a competitive mechanism (13). With respect to lipoxygenase metabolism, 20:3(n-9) is metabolized to leukotriene $A_{3}$ and subsequently leukotriene $C_{3}(18)$, but is not metabolized significantly to leukotriene $B_{3}(40)$. As with leukotriene $A_{5}$, leukotriene $A_{3}$ is poor substrate for leukotriene $A$ hydrolase and has been shown to bind to, and inactivate, leukotriene A hydrolase thus preventing leukotriene $B_{4}$ synthesis (41).

In summary, EFA deficiency has dramatic effects on both glomerular macrophage number as well as glomerular eicosanoid production. These phenomena appear to be a function solely of (n-6) fatty acids and may well be causally associated. Understanding the mechanisms underlying these observations may help to clarify further the mechanism of the protective effect of dietary fatty acid manipulation in immune-mediated glomerulonephritis. Additionally, the use of EFA deficiency may provide a new tool to elucidate the role of macrophages and arachidonate metabolism in this disorder.

\section{Acknowledgments}

The authors appreciate the technical assistance of Margery Halstead and Gary Grabau.

This study was supported by National Institutes of Health grants HL-01313 and DK-37879 (Dr. Lefkowith), and AM-36277 and a Communities Foundation of Texas grant (Dr. Schreiner).

\section{References}

1. Hurd, E. R., J. M. Johnston, J. R. Okita, P. C. MacDonald, M. Ziff, and J. N. Gilliam. 1981. Prevention of glomerulonephritis and prolonged survival in New Zealand Black/New Zealand White $F_{1}$ hybrid mice fed an essential fatty acid-deficient diet. J. Clin. Invest. 67:476-485.

2. Kelley, V. E., A. Ferretti, S. Izui, and T. B. Strom. 1985. A fish oil diet rich in eicosapentaenoic acid reduces cyclooxygenase metabolites, and suppresses lupus in MRL-lpr mice. J. Immunol. 134:19141919.

3. Prickett, J. D., D. R. Robinson, and A. D. Steinberg. 1981. Dietary enrichment with the polyunsaturated fatty acid eicosapentaenoic acid prevents proteinuria and prolongs survival in NZB $\times \mathrm{NZW} / \mathrm{F}_{1}$ mice. J. Clin. Invest. 68:556-559. 
4. Andrews, B. S., R. A. Eisenberg, A. N. Theofilopoulos, S. Izui, C. B. Wilson, P. J. McConahey, E. D. Murphy, J. B. Roths, and F. J. Dixon. 1978. Spontaneous murine lupus-like syndromes. J. Exp. Med. 148:1198-1215.

5. Schreiner, G. F., R. S. Cotran, V. Pardo, and E. R. Unanue. 1978. A mononuclear cell component in experimental immunological glomerulonephritis. J. Exp. Med. 147:369-384.

6. Holdsworth, S. R., T. J. Neale, and C. B. Wilson. 1981. Abrogation of macrophage-dependent injury in experimental glomerulonephritis in the rabbit. J. Clin. Invest. 68:686-698.

7. Stork, J. E., and M. J. Dunn. 1985. Hemodynamic roles of thromboxane $A_{2}$ and prostaglandin $E_{2}$ in glomerulonephritis. J. Pharmacol. Exp. Ther. 233:672-678.

8. Schreiner, G. F., J.-M. Kiely, R. S. Cotran, and E. R. Unanue. 1981. Characterization of resident glomerular cells in the rat expressing Ia determinants and manifesting genetically restricted interactions with lymphocytes. J. Clin. Invest. 68:920-931.

9. Schreiner, G. F., R. S. Cotran, and E. R. Unanue. 1984. Modulation of Ia and leukocyte common antigen expression in rat glomeruli during the course of glomerulonephritis and aminonucleoside nephrosis. Lab. Invest. 51:524-533.

10. Lianos, E. A., G. A. Andres, and M. J. Dunn. 1983. Glomerular prostaglandin and thromboxane synthesis in rat nephrotoxic serum nephritis. J. Clin. Invest. 72:1439-1448.

11. Lianos, E. A., M. A. Rahman, and M. J. Dunn. 1985. Glomerular arachidonate lipoxygenation in rat nephrotoxic serum nephritis. J. Clin. Invest. 76:1355-1359.

12. Lefkowith, J. B., T. Okegawa, K. DeSchryver-Kecskemeti, and P. Needleman. 1984. Macrophage-dependent arachidonate metabolism in hydronephrosis. Kidney Int. 26:10-17.

13. Lefkowith, J. B., V. Flippo, H. Sprecher, and P. Needleman. 1985. Paradoxical conservation of cardiac and renal arachidonate content in essential fatty acid deficiency. J. Biol. Chem. 260:1573615744.

14. Scharschmidt, L. A., and M. J. Dunn. 1983. Prostaglandin synthesis by rat glomerular mesangial cells in culture: effects of angiotensin II and arginine vasopressin. J. Clin. Invest. 71:1756-1764.

15. Schlondorff, D., J. A. Satriano, J. Hagege, J. Perez, and L. Baud. 1984. Effect of platelet activating factor and serum treated zymosan on $\mathrm{PGE}_{2}$ synthesis, arachidonic acid release, and contraction of cultured rat mesangial cells. J. Clin. Invest. 73:1227-1231.

16. Ardaillou, N., J. Hagege, M.-P. Nivez, R. Ardaillou, and D. Schlondorff. 1985. Vasoconstrictor-evoked prostaglandin synthesis in cultured human mesangial cells. Am. J. Physiol. 248:F240-F246.

17. Bligh, E. G., and W. J. Dyer. 1959. A rapid method of total lipid extraction and purification. Can. J. Biochem. Phsyiol. 37:911-917.

18. Lefkowith, J. B., B. A. Jakschik, P. Stahl, and P. Needleman. 1987. Metabolic and functional alterations in macrophages induced by essential fatty acid deficiency. J. Biol. Chem. 262:6668-6675.

19. Schreiner, G. F., and E. R. Unanue, 1984. Origin of the rat mesangial phagocyte and its expression of the leukocyte common antigen. Lab. Invest. 51:515-523.

20. Holman, R. 1960. The ratio of trienoic:tetraenoic aicds in tissue lipids as a measure of essential fatty acid requirement. J. Nutrition. 70:405-410.

21. Way, C. F., W. J. Dougherty, and J. A. Cook. 1985. Effects of essential fatty acid deficiency and indomethacin on histologic, ultrastructural, and phagocytic responses of hepatic macrophages to glucan. J. Leukocyte Biol. 37:137-150.

22. Kaizu, K., D. Marsh, R. Zipser, and R. J. Glassock. 1985. Role of prostaglandins and angiotensin II in experimental glomerulonephritis. Kidney Int. 28:629-635.
23. Pirotzky, E., J. Bidault, C. Burtin, M. C. Gubler, and J. Benveniste. 1984. Release of platelet-activating factor, slow-reacting substance, and vasoactive amines from isolated rat kidneys. Kidney Int. 25:404-410.

24. Schlondorff, D., J. Perez, and J. A. Satriano. 1985. Differential stimulation of $\mathrm{PGE}_{2}$ synthesis in mesangial cells by angiotensin and A23187. Am. J. Physiol. 248:C119-C126.

25. Hsueh, W., P. C. Isakson, and P. Needleman. 1977. Hormone selective lipase activation in the isolated rabbit heart. Prostaglandins. 13:1073-1091.

26. Yamanaka, W. K., G. W. Clemans, and M. L. Hutchinson. 1981. Essential fatty acid deficiency in humans. Prog. Lipid Res. 19:187-215.

27. Boissonneault, G. A., and P. V. Johnston. 1983. Essential fatty acid deficiency, prostaglandin synthesis, and humoral immunity in Lewis rats. J. Nutr. 113:1187-1194.

28. Mertin, J., and R. Hunt. 1976. Influence of polyunsaturated fatty acids on survival of skin allografts and tumor incidence in mice. Proc. Natl. Acad. Sci. USA. 73:928-931.

29. Robinson, D. R., J. D. Prickett, R. Polisson, A. D. Steinberg, and $\mathrm{L}$. Levine. 1985. The protective effect of dietary fish oil on murine lupus. Prostaglandins. 30:51-75.

30. Lefkowith, J. B., and G. F. Schreiner. 1987. Mechanisms underlying the protective effect of essential fatty acid deficiency on immune-mediated glomerulonephritis. Clin. Res. 35:565A.

31. Kelley, V. E., S. Sneve, and S. Musinski. 1986. Increased renal thromboxane production in murine lupus nephritis. J. Clin. Invest. 77:252-259.

32. Albrightson; C. R., N. L. Baenziger, and P. Needleman. 1985. Exaggerated human vascular cell prostaglandin biosynthesis mediated by monocytes: role of monokines and interleukin 1. J. Immunol. 135:1872-1877.

33. Ooi, Y. M., M. A. Weiss, A. Hsu, and B. S. Ooi. 1983. Mechanisms of suppression of mouse mesangial cell proliferation by macrophage supernatants. J. Immunol. 130:1790-1795.

34. Whitely, P. J., and P. Needleman. 1984. Mechanism of enhanced fibroblast arachidonic acid metabolism by mononuclear cell factor. J. Clin. Invest. 74:2249-2253.

35. Osborne, M. J., B. Droz, P. Meyer, and F. Morel. 1975. Angiotensin II: renal localization in glomerular mesangial cells by autoradiography. Kidney Int. 8:245-254.

36. Needleman, P., A. Raz, M. S. Minkes, J. A. Ferrendelli, and H. Sprecher. 1979. Triene prostaglandins: prostacyclin and thromboxane biosynthesis and unique biological properties. Proc. Natl. Acad. Sci. USA. 76:944-948.

37. Murphy, R. C., W. C. Pickett, B. R. Culp, and W. E. M. Lands. 1981. Tetraene and pentaene leukotrienes: selective production from murine mastocytoma cells after dietary manipulation. Prostaglandins. 22:613-622.

38. Nathaniel, D. J., J. F. Evans, Y. Leblanc, C. Leveille, B. J. Fitzsimmons, and A. W. Ford-Hutchinson. 1985. Leukotriene $A_{5}$ is a substrate and an inhibitor of rat and human neutrophil LTA $_{4}$ hydrolase. Biochem. Biophys. Res. Commun. 131:827-835.

39. Elliott, W. J., A. R. Morrison, H. Sprecher, and P. Needleman. 1986. Calcium-dependent oxidation of 5,8,11-icosatrienoic acid by the cyclooxygenase enzyme system. J. Biol. Chem. 261:6719-6724.

40. Jakschik, B. A., A. R. Morrison, and H. Sprecher. 1983. Products derived from 5,8,11-eicosatrienoic acid by the 5-lipoxygenaseleukotriene pathway. J. Biol. Chem. 258:12797-12800.

41. Evans, J. F., D. J. Nathaniel, R. J. Zamboni, and A. W. FordHutchinson. 1985. Leukotriene $A_{3}$ : a poor substrate but a potent inhibitor of rat and human neutrophil leukotriene $\mathrm{A}_{4}$ hydrolase. J. Biol. Chem. 260:10966-10970. 